Honam Mathematical J. 31 (2009), No. 1, pp. 63-73

\title{
ADJOINT SEMIGROUPS OF SUBTRACTION ALGEBRAS
}

\author{
Young Hee Kim, Kyong Ah Oh, and Tae En Jeong
}

\begin{abstract}
In this paper, we give adjoint semigroups of subtraction algebras, and investigate some properties of adjoint semigroups, and show that the adjoint semigroups of subtraction algebras are residualed semigroups.
\end{abstract}

\section{Introduction}

As a generalization of I. Fleischer [3] and T. D. Lei and C. C. Xi [9], Huang [4] introduced the notion of adjoint semigroups of BCI-algebras which establish the relationship between general BCI-algebras $[5,6]$. B. M. Schein [10] considered systems of the form $(\Phi ; \circ, \backslash)$ where $\Phi$ is a set of functions closed under the composition "o" of functions (and hence $(\Phi ; \circ)$ is a function semigroup) and the set theoretic subtraction "\" (and hence $(\Phi ; \backslash)$ is a subtraction algebra in the sense of [1]). He proved that every subtraction semigroup is isomorphic to a difference semigroup of invertible functions. B. Zelinka [12] discussed a problem proposed by B. M. Schein concerning the structure of multiplication in a subtraction semigroup. In this paper, we consider adjoint semigroups of subtration algebras, and investigate some properties of adjoint semigroup, and show that the adjoint semigroups of subtraction algebras are residualed semigroups.

\section{Preliminaries}

By a subtraction algebra ([8]), we mean an algebra $(X ;-)$ with a single binary operation "-" satisfying the following identities: for any $x, y, z \in X$,

(S1) $x-(y-x)=x$;

Received December 1, 2008. Accepted March 3, 2009.

2000 Mathematics Subject Classification. 03G25, 06B10, 06D99.

Key words and phrases: adjoint semigroup, residualed semigroup. 
(S2) $x-(x-y)=y-(y-x)$;

(S3) $(x-y)-z=(x-z)-y$.

The last identity permits us to omit parentheses in expressions of the form $(x-y)-z$. The subtraction determines an order relation on $X$ : $a \leq b \Leftrightarrow a-b=0$, where $0=a-a$ is an element which is not depend on the choice of $a \in X$. The ordered set $(X ; \leq)$ is a semi-Boolean algebra in the sense of [1], that is, it is a meet semilattice with zero 0 in which every interval $[0, a]$ is a Boolean algebra with respect to the induced order. Here $a \wedge b=a-(a-b)$; the complement of an element $b \in[0, a]$ is $a-b$; and if $b, c \in[0, a]$, then

$$
\begin{aligned}
b \vee c & =\left(b^{\prime} \wedge c^{\prime}\right)^{\prime}=a-((a-b) \wedge(a-c)) \\
& =a-((a-b)-((a-b)-(a-c))) .
\end{aligned}
$$

In a subtraction algebra, the following are true (see [8]):
(a1) $(x-y)-y=x-y$,
(a2) $x-0=x$ and $0-x=0$,
(a3) $(x-y)-x=0$,
(a4) $x-(x-y) \leq y$,
(a5) $(x-y)-(y-x)=x-y$,
(a6) $x-(x-(x-y))=x-y$,
(a7) $(x-y)-(z-y) \leq x-z$,
(a8) $x \leq y$ if and only if $x=y-w$ for some $w \in X$,
(a9) $x \leq y$ implies $x-z \leq y-z$ and $z-y \leq z-x$ for all $z \in X$,
(a10) $x, y \leq z$ implies $x-y=x \wedge(z-y)$,
(a11) $(x \wedge y)-(x \wedge z) \leq x \wedge(y-z)$.

\section{Adjoint semigroups of subtraction algebras}

For any subtraction algebra $X$ and any element $a \in X$, we denote $\rho_{a}$ the selfmap of $X$ defined by $\rho_{a}(x):=x-a$. Let $M(X)$ be the set of all finite products $\rho_{a} \cdots, \rho_{b}$ of selfmaps of $X$ with $a, \cdots, b \in X$. It is clear that $M(X)$ is a commutative monoid under multiplication of maps and $\rho_{0}$ is the identity by (S3).

We define a relation $\leq$ on $M(X)$ as follows:

$$
\rho_{u} \cdots \rho_{v} \leq \rho_{a} \cdots \rho_{b} \Longleftrightarrow \rho_{u} \cdots \rho_{v}(x)-\rho_{a} \cdots \rho_{b}(x)=0
$$

for any $x \in X$. It is easy to show that $M(X)$ becomes an ordered monoid. We call $M(X)$ an adjoint semigroup of $X$ (see [7]). For any $\sigma \in$ $M(X)$, the subsets $\operatorname{Im} \sigma:=\{\sigma(x) \mid x \in X\}, \operatorname{Ker} \sigma:=\{x \in X \mid \sigma(x)=0\}$ 
and $S(\sigma):=\{x \in X \mid \sigma(x)=x\}$ of $X$ are called the image, the kernel and stabilizer of $\sigma$ respectively (see [7]).

Example 3.1. Consider a subtraction algebra $X=\{0, a, b, c\}$ with the following Cayley table:

\begin{tabular}{c|cccc}
- & 0 & $a$ & $b$ & $c$ \\
\hline 0 & 0 & 0 & 0 & 0 \\
$a$ & $a$ & 0 & $a$ & 0 \\
$b$ & $b$ & $b$ & 0 & 0 \\
$c$ & $c$ & $b$ & $a$ & 0
\end{tabular}

Then $\rho_{0}(x)=x, \rho_{a}(x)=\left\{\begin{array}{ll}0, & \mathrm{x}=0, \mathrm{a} ; \\ b, & \mathrm{x}=\mathrm{b}, \mathrm{c}\end{array}, \rho_{b}(x)=\left\{\begin{array}{ll}0, & \mathrm{x}=0, \mathrm{~b} ; \\ a, & \mathrm{x}=\mathrm{a}, \mathrm{c}\end{array}, \rho_{c}(x)=\right.\right.$ 0 if $x$

in $X$. Thus we obtain the following table of the products of selfmaps of $X$ and $M(X)=\left\{\rho_{0}, \rho_{a}, \rho_{b}, \rho_{c}\right\}$ is an adjoint semigroup of $X$.

\begin{tabular}{c|cccc}
$\cdot$ & $\rho_{0}$ & $\rho_{a}$ & $\rho_{b}$ & $\rho_{c}$ \\
\hline$\rho_{0}$ & $\rho_{0}$ & $\rho_{a}$ & $\rho_{b}$ & $\rho_{c}$ \\
$\rho_{a}$ & $\rho_{a}$ & $\rho_{a}$ & $\rho_{c}$ & $\rho_{c}$ \\
$\rho_{b}$ & $\rho_{b}$ & $\rho_{c}$ & $\rho_{b}$ & $\rho_{c}$ \\
$\rho_{c}$ & $\rho_{c}$ & $\rho_{c}$ & $\rho_{c}$ & $\rho_{c}$
\end{tabular}

Proposition 3.2. If $X$ is a subtraction algebra, then $\operatorname{Ker} \sigma \cap S(\sigma)=$ $\{0\}$ for any $\sigma \in M(X)$.

Proof. For any $\sigma \in M(X)$,

$$
\begin{aligned}
\operatorname{Ker} \sigma \cap S(\sigma) & =\{x \in X \mid \sigma(x)=0\} \cap\{x \in X \mid \sigma(x)=x\} \\
& =\{x \in X \mid x=0\}=\{0\}
\end{aligned}
$$

Lemma 3.3. Let $X$ be a subtraction algebra. Then $\rho_{b}(a)=a$ for any $a \in X$ and $b \in \operatorname{Im} \rho_{a}$

Proof. For any $a \in X, b \in \operatorname{Im} \rho_{a}$, there exists $y \in X$ such that $b=\rho_{a}(y)=y-a$. Therefore we have $\rho_{b}(a)=a-b=a-(y-a)=a$ because of (S1). 
Lemma 3.4. [8] Every subtraction algebra satisfies the right selfdistributive law, that is, the equality $(x-y)-z=(x-z)-(y-z)$ is valid.

Definition 3.5. [8] A non-empty subset $A$ of a subtraction algebra $X$ is an ideal of $X$ if and only if it satisfies:

(I1) $0 \in A$,

(I2) $(\forall x \in X)(\forall y \in A)(x-y \in A \Rightarrow x \in A)$.

Theorem 3.6. Let $X$ be a subtraction algebra and let $M(X)$ be the adjoint semigroup of $X$. Then

1. for any $\sigma \in M(X)$ and any $a \in X, \sigma$ is an endomorphism of $X$ and $\operatorname{Im} \rho_{a}$ is an ideal of $X$,

2. $\operatorname{Ker} \rho_{a} \cap \operatorname{Im} \rho_{a}=\{0\}$ for all $a \in X$,

3. $\operatorname{Im} \sigma=S(\sigma)$ and $\operatorname{Im} \sigma$ is an ideal of $X$ for any $\sigma \in M(X)$,

4. $\operatorname{Im} \rho_{a}=S\left(\rho_{a}\right)$ and $\operatorname{Im} \rho_{a}$ is an ideal of $X$ for any $a \in X$,

5. Ker $\sigma$ is an ideal of $X$ for any $\sigma \in M(X)$,

6. $\operatorname{Ker} \rho_{a}$ is an ideal of $X$ for any $a \in X$.

3.4 ,

Proof. (1) For any $\sigma=\rho_{u} \cdots \rho_{v} \in M(X)$ and $x, y \in X$ by Lemma

$$
\begin{aligned}
\sigma(x-y) & =(\cdots((x-y)-v)-\cdots)-u \\
& =(\cdots((x-v)-(y-v))-\cdots)-u=\cdots \\
& =((\cdots(x-v)-\cdots)-u)-((\cdots(y-v)-\cdots)-u) \\
& =\sigma(x)-\sigma(y)
\end{aligned}
$$

This shows that $\sigma$ is an endomorphism. Moreover, for any $a \in X$ and $y, x-y \in \operatorname{Im} \rho_{a}, \rho_{y}(a)=a$ and $\rho_{x-y}(a)=a$ by Lemma 3.3. Hence we get,

$$
\begin{aligned}
\rho_{x}(a) & =\rho_{x}\left(\rho_{y}(a)\right)=\rho_{x} \rho_{y}(a) \\
& =\rho_{y} \rho_{x}(a)=\rho_{y}(a-x) \\
& =\rho_{y}(a)-\rho_{y}(x)=a-(x-y) \\
& =\rho_{x-y}(a)=a
\end{aligned}
$$

This leads to $x=x-(a-x)=x-\rho_{x}(a)=x-a=\rho_{a}(x)$, this is, $x \in \operatorname{Im} \rho_{a}$. Therefore, $\operatorname{Im} \rho_{a}$ is an ideal of $X$.

(2) We need only to prove that $\operatorname{Ker} \rho_{a} \cap \operatorname{Im} \rho_{a}=\{0\}$ for any $a \in X$. In fact, $0 \in \operatorname{Ker} \rho_{a} \cap \operatorname{Im} \rho_{a}$. If $x \in \operatorname{Ker} \rho_{a} \cap \operatorname{Im} \rho_{a}$, then we have $\rho_{a}(x)=0$ and $x=\rho_{a}(y)$ for some $y \in X$. Since $\rho_{a}$ is an endomorphism of $X$, we know that $x=\rho_{a}(y)=\rho_{a}(y)-\rho_{a}(a)=\rho_{a}(y-a)=\rho_{a} \rho_{a}(y)=\rho_{a}(x)=0$. Thus $\operatorname{Ker} \rho_{a} \cap \operatorname{Im} \rho_{a}=\{0\}$. 
(3) Suppose $\sigma=\rho_{u} \cdots \rho_{v} \in M(X)$ with $u, \cdots, v \in X$. Then $\sigma$ is an endomorphism of $X$ by (1), and $\sigma(u)=\rho_{u} \cdots \rho_{v}(u)=(\cdots(u-v)-$ $\cdots)-u=(\cdots(u-u)-\cdots)-v=0, \cdots, \sigma(v)=0$ by the same method. If $x \in \operatorname{Im} \sigma$ with $x=\sigma(y)$ for some $y \in X$, then

$$
\begin{aligned}
\sigma(x) & =\sigma(\sigma(y))=\sigma((\cdots(y-v)-\cdots)-u) \\
& =\sigma(\cdots(y-v)-\cdots)-\sigma(u) \\
& =(\cdots(\sigma(y)-\sigma(v))-\cdots)-\sigma(u) \\
& =\sigma(y) \\
& =x,
\end{aligned}
$$

because $\sigma(u)=\cdots=\sigma(v)=0$ and $\sigma(y)-0=\sigma(y)$. Therefore $x \in S(\sigma)$, that is, $\operatorname{Im} \sigma \subseteq S(\sigma)$. On the other hand, the conclusion $S(\sigma) \subseteq \operatorname{Im} \sigma$ is trivial. Thus $\operatorname{Im} \sigma=S(\sigma)$. Now it suffices to prove that $S(\sigma)$ is an ideal of $X$. Since $0=\sigma(0)$, we have $0 \in S(\sigma)$. If $x-y, y \in S(\sigma)$, that is, $\sigma(x-y)=x-y, \sigma(y)=y$, then $\sigma(y-x)=\rho_{u} \cdots \rho_{v}(y-x)=$ $(\cdots((y-x)-u)-\cdots)-v=((\cdots(y-u)-\cdots)-v)-x=\sigma(y)-x=y-x$, and

$$
\begin{aligned}
x-\sigma(x) & =(x-(y-x))-\sigma(x) \\
& =(x-\sigma(y-x))-\sigma(x) \\
& =(x-(\sigma(y)-\sigma(x)))-\sigma(x) \\
& =(x-\sigma(x))-(y-\sigma(x)) \\
& =(x-y)-\sigma(x) \\
& =\sigma(x-y)-\sigma(x) \\
& =(\sigma(x)-\sigma(y))-\sigma(x) \\
& =(\sigma(x)-\sigma(x))-\sigma(y) \\
& =0 .
\end{aligned}
$$

Since $\sigma \in M(X), \exists a, \cdots, b \in X$ such that $\sigma=\rho_{a} \cdots \rho_{b}$. Thus $\sigma(x)-x=$ $\left.\rho_{x}(\sigma(x))=\rho_{x}\left(\rho_{a} \cdots \rho_{b}(x)\right)\right)=((\cdots(x-b)-\cdots)-a)-x=0$ by (S3). Therefore $S(\sigma)$ is an ideal of $X$.

(4) A special case of (3).

(5) It is clear that $0 \in \operatorname{Ker} \sigma$. If $x-y, y \in \operatorname{Ker} \sigma$, that is, $\sigma(x-y)=$ $0, \sigma(y)=0$ then $\sigma(x)=\sigma(x)-0=\sigma(x)-\sigma(y)=\sigma(x-y)=0$. Hence $x \in \operatorname{Ker} \sigma$. Therefore $\operatorname{Ker} \sigma$ is an ideal of $X$.

(6) It is trivial.

A monoid is an algebra $(S ; \cdot, 1)$ with a binary operation $\cdot$ and an identity 1 satisfying : for any $x, y, z \in S$,

(m1) $x \cdot(y \cdot z)=(x \cdot y) \cdot z$, (m2) $x \cdot 1=1 \cdot x=x$.

Suppose $\leq$ is a partial ordering on $S$ such that for any $x, y, z \in S$, 
(po) $y \leq z$ implies $x \cdot y \leq x \cdot z$ and $y \cdot x \leq z \leq x$.

Then $(S ; \leq, \cdot, 1)$ is called to be a partially ordered monoid(briefly pomonoid). And a pomonoid $(S ; \leq, \cdot, 1)$ is called to be commutative if it satisfies

$$
x \cdot y=y \cdot x,
$$

for any $x, y \in S$. Let $(S, \cdot, \leq)$ be a commutative pomonoid. By the residual $a: b$, we mean a greatest element $x$ (if it exists) such that $b \cdot x \leq a$. We now call $S$ a residualed semigroup if $a: b$ exists for all $a, b \in S$. It is easy to prove that $a: b \cdot c=(a: b): c($ see$[2,3])$.

Lemma 3.7. Let $X$ be a subtraction algebra and $M(X)$ be the adjoint semigroup of $X$. Then $\rho_{a} \leq \rho_{b}$ in $M(X)$ if and only if $b \leq a$, and $\rho_{a}=\rho_{b}$ if and only if $a=b$ for any $a, b \in X$.

Proof. If $\rho_{a} \leq \rho_{b}$ then $b-a=(b-a)-(b-b)=\rho_{a}(b)-\rho_{b}(b)=0$, that is, $b \leq a$. On the other hand, if $b \leq a$ then $\rho_{a}(x)-\rho_{b}(x)=$ $(x-a)-(x-b) \leq b-a=0$ for all $x \in X$. Therefore $\rho_{a} \leq \rho_{b}$. It is trivial that $a=b$ implies $\rho_{a}=\rho_{b}$. If $\rho_{a}=\rho_{b}$ then $a-b=\rho_{b}(a)=$ $\rho_{a}(a)=0=\rho_{b}(b)=\rho_{a}(b)=b-a$, and thus $a=b$. The proof is completed.

Lemma 3.8. Let $X$ be a subtraction algebra and $M(X)$ be the adjoint semigroup of $X$. Then for any $a, b \in X, \rho_{a}$ is a residuable element of $M(X)$ and $\rho_{a}: \rho_{b}=\rho_{a-b}$, i.e, $\rho_{a}: \rho_{b}=\sup \left\{\rho_{c} \in M(X) \mid \rho_{b} \rho_{c} \leq \rho_{a}\right\}$.

Proof. Given $x \in X$, we obtain

$$
\begin{aligned}
\rho_{b} \rho_{a-b}(x) & =\rho_{b}(x-(a-b)) \\
& =(x-(a-b))-b \\
& =(x-b)-(a-b) \\
& \leq x-a \\
& =\rho_{a}(x) .
\end{aligned}
$$

Hence we have

$$
\rho_{b} \rho_{a-b} \leq \rho_{a}
$$

To show $\rho_{a-b}$ is the greatest element of $M(X)$ satisfying (3.1), we suppose that $\rho_{b} \sigma \leq \rho_{a}$ for some $\sigma \in M(X)$. Assume $\sigma=\rho_{a_{1}} \cdots \rho_{a_{n}}$ for some 
$a_{1}, \cdots, a_{n} \in X$. Then $\rho_{b} \sigma(a)=\rho_{b} \rho_{a_{1}} \cdots \rho_{a_{n}}(a) \leq \rho_{a}(a)=a-a=0$, and hence $\rho_{b} \sigma(a)=\rho_{b} \rho_{a_{1}} \cdots \rho_{a_{n}}(a)=0$. Thus for any $x \in X$, we have

$$
\begin{aligned}
\rho_{a_{1}} \cdots \rho_{a_{n}}(x)-\rho_{a-b}(x) & =\left(\left(\cdots\left(x-a_{n}\right)-\cdots\right)-a_{1}\right)-(x-(a-b)) \\
& =\left(\cdots\left((x-(x-(a-b)))-a_{n}\right)-\cdots\right)-a_{1} \\
& \leq\left(\cdots\left((a-b)-a_{n}\right)-\cdots\right)-a_{1} \\
& =\left(\left(\cdots\left(a-a_{n}\right)-\cdots\right)-a_{1}\right)-b \\
& =\rho_{b} \rho_{a_{1}} \cdots \rho_{a_{n}}(a) \\
& =0 .
\end{aligned}
$$

Hence

$$
\sigma=\rho_{a_{1}} \cdots \rho_{a_{n}} \leq \rho_{a-b} .
$$

From (3.1) and (3.2), we obtain $\rho_{a}: \rho_{b}=\rho_{a-b}$.

Example 3.9. Consider a subtraction algebra $X:=\{0, a, b, c, d\}$ with the following Cayley table:

\begin{tabular}{c|ccccc}
- & 0 & $a$ & $b$ & $c$ & $d$ \\
\hline 0 & 0 & 0 & 0 & 0 & 0 \\
$a$ & $a$ & 0 & $a$ & 0 & $a$ \\
$b$ & $b$ & $b$ & 0 & 0 & $b$ \\
$c$ & $c$ & $b$ & $a$ & 0 & $c$ \\
$d$ & $d$ & $d$ & $d$ & $d$ & $d$
\end{tabular}

Let $M(X)$ be the adjoint semigroup of $X$. Put $\sigma=\rho_{a} \rho_{b}$ and $\tau=\rho_{c} \rho_{d}$ Then $\sigma=\rho_{c}$ and $\tau=0$. Since $\sigma: \tau=\sup \{\nu \in M(X) \mid \sigma \nu \leq \tau\}$, there exists $\sigma: \tau=\rho_{d}$.

Theorem 3.10. Let $X$ be a subtraction algebra and $M(X)$ be an adjoint semigroup of $X$. For any $\sigma, \tau \in M(X)$, if $\sigma: \tau$ exists, then we denote that

$$
\sigma-\tau=\sigma: \tau .
$$

Then $\rho_{a}-\rho_{b}=\rho_{a-b}$ for any $a, b \in X$ and the following statements hold:

1. $\sigma-\rho_{0}=\sigma$,

2. $\sigma-\sigma=\rho_{0}$,

3. $\rho_{0}-\sigma=\rho_{0}$.

Proof. It follows from Lemma 3.8 that $\rho_{a}-\rho_{b}=\rho_{a-b}$.

(1) Since $\rho_{0} \sigma=\sigma$ for any $\sigma \in M(X), \sigma-\rho_{0}=\sigma$.

(2) Since $\sigma \rho_{0}=\sigma$ for any $\sigma \in M(X), \sigma-\sigma=\rho_{0}$.

(3) Since $\sigma \leq \rho_{0}$ for any $\sigma \in M(X), \sigma \rho_{0}=\sigma \leq \rho_{0}$, which proves $\rho_{0}-\sigma=\rho_{0}$. 
Theorem 3.11. Let $X$ be a subtraction algebra and $M(X)$ be an adjoint semigroup of $X$. For any $a, b \in X$, we define '-' on $M(X)$ such that $\rho_{a}-\rho_{b}=\rho_{a}: \rho_{b}$. Then the following statements hold:

1. $\rho_{a}-\rho_{0}=\rho_{a}$ implies $\rho_{a}=\rho_{0}$,

2. $\rho_{a}-\rho_{a}=\rho_{0}$,

3. $\left(\rho_{a}-\left(\rho_{a}-\rho_{b}\right)\right)-\rho_{b}=\rho_{0}$,

4. $\left(\left(\rho_{a}-\rho_{b}\right)-\left(\rho_{a}-\rho_{c}\right)\right)-\left(\rho_{c}-\rho_{b}\right)=\rho_{0}$.

Proof. By Lemma 3.8 and Theorem 3.10.

Lemma 3.12. Let $X$ be a subtraction algebra and $M(X)$ be an adjoint semigroup of $X$. Then $\rho_{a}-\sigma$ exists in $M(X)$ and $\rho_{a}-\sigma=\rho_{\sigma(a)}$ for $\sigma \in M(X)$ and $a \in X$.

Proof. For all $x \in X$, we have

$$
\begin{aligned}
\sigma \rho_{\sigma(a)}(x) & =\sigma(x-\sigma(a)) \\
& =\sigma(x)-\sigma \sigma(a) \\
& =\sigma(x)-\sigma(a) \\
& =\sigma(x-a) \\
& \leq \rho_{0}(x-a) \\
& =\rho_{0} \rho_{a}(x) \\
& =\rho_{a}(x)
\end{aligned}
$$

by Theorem 3.6. It follows that

$$
\sigma \rho_{\sigma(a)} \leq \rho_{a} .
$$

To show $\rho_{\sigma(a)}$ is the greatest element of $M(X)$ satisfying (3.3), we suppose that $\sigma \tau \leq \rho_{a}$ for some $\tau \in M(X)$. Assume $\tau=\rho_{a_{1}} \cdots \rho_{a_{n}}$ for some $a_{1}, \cdots, a_{n} \in X$. Then $\sigma \tau(a)=\sigma \rho_{a_{1}} \cdots \rho_{a_{n}}(a) \leq \rho_{a}(a)=a-a=0$, and hence $\sigma \tau(a)=\sigma \rho_{a_{1}} \cdots \rho_{a_{n}}(a)=0$. Thus for any $x \in X$, we have

$$
\begin{aligned}
\tau(x)-\rho_{\sigma(a)}(x) & =\rho_{a_{1}} \cdots \rho_{a_{n}}(x)-\rho_{\sigma(a)}(x) \\
& \left.=\left(\cdots\left(x-a_{n}\right)-\cdots\right)-a_{1}\right)-(x-\sigma(a)) \\
& =\left(\cdots\left((x-(x-\sigma(a)))-a_{n}\right)-\cdots\right)-a_{1} \\
& \leq\left(\cdots\left(\sigma(a)-a_{n}\right)-\cdots\right)-a_{1} \\
& =\rho_{a_{1}} \cdots \rho_{a_{n}}(\sigma(a)) \\
& =\sigma \rho_{a_{1}} \cdots \rho_{a_{n}}(a) \\
& =0
\end{aligned}
$$

because $M(X)$ is commutative. Thus

$$
\tau=\rho_{a_{1}} \cdots \rho_{a_{n}} \leq \rho_{\sigma(a)} .
$$


From (3.3) and (3.4), we obtain $\rho_{a}-\sigma=\rho_{\sigma(a)}$.

Lemma 3.13. Let $X$ be a subtraction algebra. Then $\sigma-\rho_{c}$ exists for any $\sigma, \rho_{c} \in M(X)$. Furthermore if $\sigma=\rho_{a_{1}} \cdots \rho_{a_{m}}$ for some $a_{1}, \cdots, a_{n} \in$ $X$, then $\sigma-\rho_{c}=\rho_{a_{1}-c} \cdots \rho_{a_{m}-c}$.

Proof. Let $\sigma=\rho_{a_{1}} \cdots \rho_{a_{m}}$ for some $a_{1}, \cdots a_{m} \in X$. For all $x \in X$, by Lemma 3.4 and (a9), we have

$$
\begin{aligned}
\rho_{c} \rho_{a_{1}-c} \cdots \rho_{a_{m}-c}(x) & =\rho_{a_{1}-c} \cdots \rho_{a_{m}-c} \rho_{c}(x) \\
& =\left(\cdots\left((x-c)-\left(a_{m}-c\right)\right)-\cdots\right)-\left(a_{1}-c\right) \\
& =\left(\cdots\left(\left(x-a_{m}\right)-c\right)-\cdots\right)-\left(a_{1}-c\right) \\
& =\cdots \\
& =\left(\left(\cdots\left(x-a_{m}\right)-\cdots\right)-a_{1}\right)-c \\
& \leq\left(\cdots\left(x-a_{m}\right)-\cdots\right)-a_{1} \\
& =\rho_{a_{1}} \cdots \rho_{a_{m}}(x) .
\end{aligned}
$$

It follows that

$$
\rho_{c} \rho_{a_{1}-c} \cdots \rho_{a_{m}-c} \leq \rho_{a_{1}} \cdots \rho_{a_{m}} .
$$

Moreover, if $\tau=\rho_{b_{1}} \cdots \rho_{b_{n}} \in M(X)$ such that $\rho_{c} \tau \leq \sigma$, then $\rho_{c} \tau(x)-$ $\sigma(x)=0$ for any $x \in X$. In particular, we have $\tau\left(a_{1}-c\right)=\cdots=$ $\tau\left(a_{m}-c\right)=0$ because if $\rho_{c} \tau=\tau \rho_{c}$ and $\sigma\left(a_{i}\right)=0, \forall i=1,2, \cdots, m$. Hence

$$
\begin{aligned}
& \tau(x)-\rho_{a_{1}-c} \cdots \rho_{a_{m}-c}(x) \\
& =\left(\left(\cdots\left(x-b_{n}\right)-\cdots\right)-b_{1}\right)-\left(\left(\cdots\left(x-\left(a_{m}-c\right)\right)-\cdots\right)-\left(a_{1}-c\right)\right) \\
& =\left(\cdots\left(\left(x-\left(\left(\cdots\left(x-\left(a_{m}-c\right)\right)-\cdots\right)-\left(a_{1}-c\right)\right)\right)-b_{n}\right)-\cdots\right)-b_{1} \\
& =\tau\left(x-\left(\left(\cdots\left(x-\left(a_{m}-c\right)\right)-\cdots\right)-\left(a_{1}-c\right)\right)\right. \\
& =\tau(x)-\tau\left(\left(\cdots\left(x-\left(a_{m}-c\right)\right)-\cdots\right)-\left(a_{1}-c\right)\right) \\
& =\tau(x)-\left(\left(\cdots\left(\tau(x)-\tau\left(a_{m}-c\right)\right)-\cdots\right)-\tau\left(a_{1}-c\right)\right) \\
& =\tau(x)-\tau(x) \\
& =0
\end{aligned}
$$

for all $x \in X$. Hence

$$
\tau \leq \rho_{a_{1}-c} \cdots \rho_{a_{m}-c} .
$$

From (3.5) and (3.6), we obtain $\sigma-\rho_{c}=\rho_{a_{1}-c} \cdots \rho_{a_{m}-c}$.

Theorem 3.14. Let $X$ be a subtraction algebra. Then $M(X)$ is a residualed semigroup with $\left(\rho_{a}-\rho_{b}\right)-\rho_{c}=\left(\rho_{a}-\rho_{c}\right)-\left(\rho_{b}-\rho_{c}\right)$ and $\rho_{a}-\left(\rho_{a}-\rho_{b}\right)=\rho_{b}-\left(\rho_{b}-\rho_{a}\right)$. 
Proof. For any $\sigma=\rho_{a_{1}} \cdots \rho_{a_{m}}=\rho_{a_{m}} \cdots \rho_{a_{1}}, \tau=\rho_{b_{1}} \cdots \rho_{b_{n}}=\rho_{b_{n}} \cdots \rho_{b_{1}} \in$ $M(X), \sigma-\rho_{b_{1}},\left(\sigma-\rho_{b_{1}}\right)-\rho_{b_{2}}, \cdots$ and $\left(\cdots\left(\left(\sigma-\rho_{b_{1}}\right)-\rho_{b_{2}}\right)-\cdots\right)-\rho_{b_{n}}$ exist by Lemma 3.13 and therefore $\sigma-\tau$ exists. Since $\rho_{a}-\rho_{b} \rho_{c}=$ $\rho_{\rho_{b} \rho_{c}(a)}=\rho_{(a-c)-b}=\rho_{(a-b)-c}=\rho_{a-b}-\rho_{c}=\left(\rho_{a}-\rho_{b}\right)-\rho_{c}$ holds in $M(X)$ by Lemma 3.12, we obtain that

$$
\begin{aligned}
\sigma-\tau & =\sigma-\rho_{b_{1}} \cdots \rho_{b_{n}} \\
& =\left(\cdots\left(\sigma-\rho_{b_{n}}\right)-\cdots\right)-\rho_{b_{1}} \\
& =\left(\cdots\left(\rho_{a_{1}} \cdots \rho_{a_{m}}-\rho_{b_{n}}\right)-\cdots\right)-\rho_{b_{1}} \\
& =\left(\cdots\left(\rho_{a_{1}-b_{n}} \cdots \rho_{a_{m}-b_{n}}\right)-\cdots\right)-\rho_{b_{1}} \\
& =\cdots \\
& =\rho_{\left(\cdots\left(a_{1}-b_{n}\right)-\cdots\right)-b_{1}} \cdots \rho_{\left(\cdots\left(a_{m}-b_{n}\right)-\cdots\right)-b_{1}} \\
& =\rho_{\tau\left(a_{1}\right)} \cdots \rho_{\tau\left(a_{m}\right)}
\end{aligned}
$$

where $\tau\left(a_{1}\right)=\left(\cdots\left(a_{1}-b_{n}\right)-\cdots\right)-b_{1}, \cdots, \tau\left(a_{m}\right)=\left(\cdots\left(a_{m}-b_{n}\right)-\right.$ $\cdots)-b_{1}$. Hence $M(X)$ is a residualed semigroup. Moreover, we can see that

$$
\begin{aligned}
\left(\rho_{a}-\rho_{b}\right)-\rho_{c} & =\rho_{a-b}-\rho_{c} \\
& =\rho_{(a-b)-c} \\
& =\rho_{(a-c)-(b-c)} \\
& =\rho_{a-c}-\rho_{b-c} \\
& =\left(\rho_{a}-\rho_{c}\right)-\left(\rho_{b}-\rho_{c}\right),
\end{aligned}
$$

and

$$
\begin{aligned}
\rho_{a}-\left(\rho_{a}-\rho_{b}\right) & =\rho_{a}-\rho_{a-b} \\
& =\rho_{a-(a-b)} \\
& =\rho_{b-(b-a)} \\
& =\rho_{b}-\rho_{b-a} \\
& =\rho_{b}-\left(\rho_{b}-\rho_{a}\right) .
\end{aligned}
$$

\section{References}

[1] J. C. Abbott, Sets, lattices and Boolean algebras, Allyn and Bacon, Boston, 1969.

[2] G. Birkhoff, Lattice Theory, Amer. Math. Soc. Colloq. Publ., Vol. 25, second edition 1984; third edition, 1967, Providence.

[3] I. Fleischer, Every BCK-algebra is a set of residuables in an integral pomonoid, J. Algebra 119(1988), 360-365.

[4] W. P. Huang, On BCI-algeberas and semigroups, Math. Japon. 42(1995), 59-64.

[5] W. P. Huang, Residualed semigroups and BCI-algebras with condition(S), Math. Japon. 43(1996), 545-548.

[6] W. P. Huang and F. Liu, On the adjoint semigroups of p-separable BCI-algebras, Semigroup Forum 58(1999), 317-322. 
[7] W.P. Huang and D.J. Sun, Periodic BCI-algebras of adjoint monoids of BCIalgebras, Semigroup Forum 57(1998), 315-320.

[8] Y. B. Jun, H. S. Kim and E. H. Roh, Ideal theory of subtraction algebras, Sci. Math. Jpn. 61 (2005), no. 3, 459-464, :e-2004, 397-402.

[9] T. D. Lei and C. C. Xi, p-radical in BCI-algebras, Math. Japon. 30(1985), 511517.

[10] B. M. Schein, Difference semigroups, Comm. Algebra 20(1992), 2153-2169.

[11] S. Xu, On the adjoint monoids of implicative BCK-algebras, Southeast Asian Bulletin of Mathematics 26(2002), 535-540.

[12] B. Zelinka, Subtraction semigroups, Math. Bohemica 120 (1995), 445-447.

Young Hee Kim

Department of Mathematics

Chungbuk National University

Cheongju 361-763, Korea

Email : yhkim@cbnu.ac.kr

\section{Kyong Ah Oh}

Department of Mathematics

Chungbuk National University

Cheongju 361-763, Korea

Email : kyongahoh@hanmail.net

Tae En Jeong

Department of Mathematics

Chungbuk National University

Cheongju 361-763, Korea

Email : algetae@hanmail.net 